POS $\quad \begin{aligned} & \text { PROCEEDINGS } \\ & \text { OF SCIENCE }\end{aligned}$

\title{
Quantization of the symplectic groupoid
}

\author{
F. Bonechi* \\ INFN Sezione di Firenze \\ E-mail: bonechidfi,infn.it \\ N. Ciccoli \\ Dipartimento di Matematica, Univ. di Perugia \\ E-mail: Ciccoliddipmat.unipg.it
}

\section{M.Tarlini}

INFN Sezione di Firenze

E-mail: tarliniefi.infn.it

We present the geometric quantization of the standard Podles̀ sphere by using a multiplicative real polarization of the symplectic groupoid. We introduce the concept of multiplicative integrability of the modular function as one key point of the construction.

Proceedings of the Corfu Summer Institute 2011 School and Workshops on Elementary Particle Physics and Gravity

September 4-18, 2011

Corfu, Greece

\footnotetext{
* Speaker.
} 


\section{Introduction}

Quantization of Poisson manifolds has been the main motivation for introducing the concept of symplectic groupoid, see for instance in [12]. A symplectic groupoid is a Lie groupoid $\mathscr{G}$ with a compatible symplectic form $\Omega \mathscr{G}$. This compatibility means that the graph of the groupoid multiplication is a lagrangian submanifold of $\mathscr{G} \times \mathscr{G} \times \overline{\mathscr{G}}$, where $\overline{\mathscr{G}}$ means $\mathscr{G}$ endowed with $-\Omega \mathscr{G}$. These data allow to define a Poisson tensor on the submanifold of unities; the converse procedure of associating a symplectic groupoid to a given Poisson manifold is the analogue of integrating a Lie algebra and shares with Lie theory important properties. For this reason a Poisson manifold that is the space of unities of a symplectic groupoid is called integrable. According to the general paradigma of quantization coming from semiclassical analysis (see [四]), lagrangian submanifolds correspond to states of the Hilbert space, so that the quantization of the symplectic groupoid should produce a given state of the triple tensor product, that endows the Hilbert space of states of an algebra structure. This algebra must be seen as the quantization of the Poisson manifold.

Since then, the role of symplectic groupoids in the study of Poisson geometry has been more and more relevant, but the original program of quantization remained in the background. The main reason for this loss of interest lies in the intrinsic limitations of geometric quantization which is the main tool for quantization. Indeed, there is a canonical procedure only for quantizing cotangent bundles and Kahler manifolds, but the vertical polarization of the cotangent bundle is compatible only with the trivial Poisson structure and a Kahler structure is not typical for symplectic groupoids. As a result, the only concrete advancement has been the understanding of prequantization given in [144] and the only worked out example has been that of irrational rotations on the torus in [ए33].

Very recently, E. Hawkins in [U] revived the subject. According to his proposal, geometric quantization should produce a $C^{*}$-algebra on the space of states. A key concept is that of multiplicative polarization, i.e. a polarization which is compatible with the groupoid structure, such that a convolution product can be defined on the space of polarized sections. Since a multiplicative polarization is an ordinary polarization that is compatible with the groupoid structures, the main difficulties of geometric quantization are still there. In [ $[0]$ it is observed that the ideal case of multiplicative real polarization is given by a groupoid fibration, i.e. the space of leaves is itself a groupoid and the convolution algebra should be in that case its convolution algebra.

Our starting observation, is that thanks to the groupoid structure the usual path of geometric quantization can be abandoned and resort to the theory of groupoid $C^{*}$-algebras, introduced by $\mathrm{J}$. Reanult in [Q] . Indeed, the regularity that we have to demand is that the groupoid of leaves admits an Haar system, i.e. a way of integrating along the fibres of the range map. With this structure at hand, one can apply Renault's construction and get the convolution algebra as the quantization of the Poisson manifold. In order to do this, we can consider real polarization with singularities much more severe than those allowed by geometric quantization. If the space of leaves is a Lie groupoid then there is a canonical Haar system but in this case one needs the usual regularity conditions for real polarization and the construction is the same as definining a convolution product on the space of sections. Remarkably, another favourable situation is when the foliation admits BohrSommerfeld (BS) selection rules and the groupoid of BS-leaves is for instance $r$-discrete, i.e. it has discrete range fibres. In this case the foliation can be rather crazy from the point of view of smooth structure, even not anymore a distribution, but one can still apply Renault theory instead of 
geometric quantization.

One important ingredient is the quantization of the modular function. This function integrates the modular vector field, introduced in [15] as an invariant measuring the obstruction to have a volume form invariant with respect to the flow of hamiltonian vector fields. Most importantly, it is the semicalssical analogue of the Tomita-Takesaki operator in Von Neumann and $C^{*}$-algebras. Every quantization procedure should make them correspond and we have to require that our polarization allows the quantization of the modular function. It is tempting then to discuss the real singular polarization in terms of integrability of the modular function. This brings us to formulate the concept of multiplicative integral model.

There is an interesting class of examples where one can test these ideas, Poisson Lie groups and their homogeneous spaces. Indeed, in a series of papers A. Sheu (see [ए, एW]) showed that the $C^{*}$-algebra of a compact quantum groups can be realized as a subalgebra of a groupoid $C^{*}$-algebra. We claim that this groupoid appears as groupoid of Bohr-Sommerfeld leaves of some multiplicative integrable model on the symplectic groupoid of the underlying Poisson manifold. This project started from the simplest Poisson homogeneous space of Poisson Lie groups, the Podles̀ sphere in [ㅁ] , and will be continued in [B]]. In this short note we present the case of the Podles̀ sphere studied in [■] from the point of view of integrable models.

\section{Symplectic groupoids}

Let $\mathscr{G}=\left(\mathscr{G}, \mathscr{G}_{0}, l_{\mathscr{G}}, r_{\mathscr{G}}, m_{\mathscr{G}}, l_{\mathscr{G}}, \mathcal{E}_{\mathscr{G}}\right)$ be a groupoid over the space of units $\mathscr{G}_{0}$, where $l_{\mathscr{G}}, r_{\mathscr{G}}: \mathscr{G} \rightarrow$ $\mathscr{G}_{0}$ are the source and target maps, respectively, $m_{\mathscr{G}}: \mathscr{G}_{2} \rightarrow \mathscr{G}$ is the multiplication, $\iota_{\mathscr{G}}: \mathscr{G} \rightarrow \mathscr{G}$ is the inversion and $\varepsilon_{\mathscr{G}}: \mathscr{G}_{0} \rightarrow \mathscr{G}$ is the embedding of units. Our conventions are that $\left(\gamma_{1}, \gamma_{2}\right) \in \mathscr{G}_{2}$ if $r_{\mathscr{G}}\left(\gamma_{1}\right)=l_{\mathscr{G}}\left(\gamma_{2}\right)$. We will denote $m_{\mathscr{G}}\left(\gamma_{1}, \gamma_{2}\right)=\gamma_{1} \gamma_{2}$. We say that $\mathscr{G}$ is source simply connected (ssc) if $l_{\mathscr{G}}^{-1}(x)$ is connected and simply connected for any $x \in \mathscr{G}_{0}$. A real function $f \in C(\mathscr{G})$ is 1-cocycle if $f\left(\gamma_{1} \gamma_{2}\right)=f\left(\gamma_{1}\right)+f\left(\gamma_{2}\right)$, for $\left(\gamma_{1}, \gamma_{2}\right) \in \mathscr{G}_{2}$; we denote the set of real 1-cocycles as $Z^{1}(\mathscr{G}, \mathbb{R})$.

A groupoid is Lie when $\mathscr{G}$ and $\mathscr{G}_{0}$ are both smooth manifolds, all structure maps are smooth maps and $l_{\mathscr{G}}, r_{\mathscr{G}}$ are surjective submersions. A symplectic groupoid is a Lie groupoid, which is equipped with a symplectic form $\Omega \mathscr{G}$, such that the graph of the multiplication is a lagrangian submanifold of $\mathscr{G} \times \mathscr{G} \times \overline{\mathscr{G}}$, where $\overline{\mathscr{G}}$ means $\mathscr{G}$ with the opposite symplectic structure. An equivalent characterization for a Lie groupoid $\mathscr{G}$ to be a symplectic groupoid is that the symplectic form be multiplicative, i.e. $\partial^{*}\left(\Omega_{\mathscr{G}}\right)=\left(m_{\mathscr{G}}^{*}-\mathrm{pr}_{1}^{*}-\mathrm{pr}_{2}^{*}\right) \Omega_{\mathscr{G}}=0$, where $\mathrm{pr}_{i}: \mathscr{G}_{2} \subset \mathscr{G} \times \mathscr{G} \rightarrow \mathscr{G}$ is the projection on the $i$-th factor. There exists a unique Poisson structure on $\mathscr{G}_{0}$ such that $l_{\mathscr{G}}$ and $r_{\mathscr{G}}$ are Poisson and anti-Poisson mappings, respectively. A Poisson manifold is said to be integrable if it is the space of units of a symplectic groupoid.

Let $(M, \pi)$ be a Poisson manifold, where we denote with $\pi$ the bivector defined by the Poisson bracket as $\{f, g\}=\pi^{\mu v} \partial_{\mu}(f) \partial_{v}(g)$. As a consequence of the Jacobi identity of the Poisson brackets, $d_{L P}(X)=[\pi, X]$ squares to zero; its cohomology is called the Lichnerowicz-Poisson cohomology and is denoted with $H_{L P}(M, \pi)$. Two distinguished classes are relevant for what follows. The first one is the class $[\pi] \in H_{L P}^{2}(M, \pi)$ defined by the Poisson tensor itself. Let us assume that $M$ is orientable, and let us choose a volume form $V_{M}$ on $M$. The modular vector field $\chi_{V_{M}}=\operatorname{div}_{V_{M}} \pi$ 
is $d_{L P}$-closed; its cohomology class $\chi_{V_{M}} \in H_{L P}^{1}(M, \pi)$ does not depend on the choice of the volume form and is called the modular class.

We can always associate to a Poisson manifold $(M, \pi)$ a topological groupoid $\Sigma(M)$ [䧃, 目]. The elements of $\Sigma(M)$ are equivalence classes of cotangent paths under the cotangent homotopy. A cotangent path is a $C^{1}$-path $c: I \rightarrow T^{*} M$ such that

$$
\pi^{\sharp}(c(t))=\frac{d}{d t} p(c(t)),
$$

with $p: T^{*} M \rightarrow M$ is the cotangent projection and the sharp map $\pi^{\sharp}: T^{*} M \rightarrow T M$ denotes the contraction with $\pi$. The source and target maps $l, r: \Sigma(M) \rightarrow M$ are given by $l([c])=p(c(0))$ and $r([c])=p(c(1))$, the multiplication in $\Sigma(M)$ is defined by the concatenation of cotangent paths. In general $\Sigma(M)$ is a topological groupoid, but if it is smooth $\Sigma(M)$ carries a symplectic structure that makes it the unique (ssc) symplectic groupoid integrating $(M, \pi)$.

Using this description of the symplectic groupoid, it is easy to see that any vector field $\chi$ of $M$ that is closed $d_{L P}(\chi)=0$ can be lifted to a groupoid 1-cocycle $F_{\chi}$ defined as

$$
F_{\chi}([c])=\int_{0}^{1}\langle\chi(p(c(t))), c(t)\rangle d t
$$

In particular the modular vector field $\chi_{V_{M}}$ is lifted to the modular function $F_{V_{M}}$.

\section{Geometric quantization of the symplectic groupoid}

Let $\mathscr{G}(M)$ be a symplectic groupoid integrating the Poisson manifold $(M, \pi)$. We recall in this section known facts about its geometric quantization.

Let us consider first the prequantization, as studied in [ए4]]. Let us assume that $\mathscr{G}(M)$ is prequantizable as symplectic manifold and let $(\Lambda, \nabla)$ be a prequantization, where $\Lambda$ is an hermitian line bundle over $\mathscr{G}(M)$ and $\nabla$ a connection satisfying $\nabla^{2}=-i / \hbar \Omega \mathscr{G}$. Let $\partial^{*} \Lambda^{*}$ denote the (hermitian) line bundle $\operatorname{pr}_{1}^{*} \Lambda^{*} \otimes \operatorname{pr}_{2}^{*} \Lambda^{*} \otimes m_{\mathscr{G}}^{*} \Lambda$ over $\mathscr{G}_{2}(M)$, where $\operatorname{pr}_{i}$ denote the projections from the $i$-th factor of $\mathscr{G}(M) \times \mathscr{G}(M)$ and $m \mathscr{G}$ the multiplication on $\mathscr{G}(M)$. We give the following definition.

Definition 3.1. A prequantization of the symplectic groupoid $\mathscr{G}(M)$ consists of the triple $(\Lambda, \nabla ; \zeta)$ where $\zeta$ is a section of $\partial^{*} \Lambda^{*}$ such that:

i) $\zeta$ has norm one and is multiplicative, i.e. it satisfies for $\left(\gamma_{1}, \gamma_{2}, \gamma_{3}\right) \in \mathscr{G}_{3}(M)$

$$
\zeta\left(\gamma_{1}, m_{\mathscr{G}}\left(\gamma_{2}, \gamma_{3}\right)\right) \otimes \zeta\left(\gamma_{2}, \gamma_{3}\right)=\zeta\left(\gamma_{1}, \gamma_{2}\right) \otimes \zeta\left(m_{\mathscr{G}}\left(\gamma_{1}, \gamma_{2}\right), \gamma_{3}\right)
$$

ii) $\zeta$ is covariantly constant, i.e. if $\Theta_{\mathscr{G}}$ is a (local) primitive of $\Omega \mathscr{G}$, then $\zeta$ (locally) satisfies

$$
d \zeta+\left(\partial^{*} \Theta_{\mathscr{G}}\right) \zeta=0
$$


In [14] it is shown that if $\mathscr{G}(M)$ is prequantizable as a symplectic manifold then there exists a unique groupoid prequantization in the sense of Definition 3. . We call $\zeta$ the prequantization cocycle; the set of two-cocycles with values in $\mathbb{T}$ is denoted as $Z^{2}(\mathscr{G}, \mathbb{T})$. It is determined by the choice of a (local) primitive $\Theta_{\mathscr{G}}$; it is shown in [可] that $\Theta_{\mathscr{G}}$ can be chosen such that $\partial^{*} \Theta_{\mathscr{G}}=0$ (and so $\zeta=1)$ if and only if $[\pi]=0$ in $H_{L P}^{2}(M, \pi)$.

The second step in geometric quantization is the choice of a polarization. We require the compatibility with the groupoid structure expressed by the following definition given in [D] in order to construct a convolution product between polarized sections.

Definition 3.2. For any distribution $\mathscr{P} \subset T_{\mathbb{C}} \mathscr{G}(M)$, let us denote $\mathscr{P}_{2}=(\mathscr{P} \times \mathscr{P}) \cap T_{\mathbb{C}} \mathscr{G}_{2}(M)$. Then $\mathscr{P}$ is called a multiplicative polarization if it is lagrangian and, for every $\left(\gamma_{1}, \gamma_{2}\right) \in \mathscr{G}_{2}(M)$,

$$
m_{\mathscr{G}}\left(\mathscr{P}_{2}\right)_{\left(\gamma_{1}, \gamma_{2}\right)}=\mathscr{P}_{m_{\mathscr{G}}\left(\gamma_{1}, \gamma_{2}\right)} .
$$

We want to discuss the special case of real polarization for the symplectic groupoid given by an integrable model, compatible with the groupoid structure.

Let $\left\{F_{i}\right\}_{i=1}^{\operatorname{dim} \mathscr{G} / 2}$ a maximal set of independent functions $F_{i} \in C^{\infty}(\mathscr{G}(M))$ in involution; i.e. we require that $\left\{F_{i}, F_{j}\right\}=0$ and that $\mathscr{P}_{\gamma}=\left\langle\chi_{F_{i}}(\gamma)\right\rangle$ has dimension $\operatorname{dim} \mathscr{G} / 2$ almost everywhere (i.e. everywhere but in a set of measure zero). These functions must be multiplicative, i.e. we require that the (singular) distribution $\mathscr{P}$ is multiplicative in the sense of definition (B.2). We require two additional properties: $i$ ) the modular function $F$, if non vanishing, must be one of the hamiltonians $F_{i}$ (say $F_{1}$ ); ii) there exists a choice of $\Theta_{\mathscr{G}}$ of the primitive of the symplectic form $\Omega_{\mathscr{G}}$ satisfying $\partial^{*} \Theta_{\mathscr{G}} \in \mathscr{P}_{2}^{\perp}$.

Let us stress that the modular function depends on the choice of a volume form on $M$. If all these conditions are fulfilled, then we say that the modular function defines a multiplicatively integrable dynamical system.

The leaves of this polarization are by construction the contour levels of the hamiltonians $F_{i}$; the Bohr-Sommerfeld leaves are those lagrangian leaves $L$ such that $\left.\left(\Theta_{\mathscr{G}}\right)\right|_{L}$ defines a class in $H^{1}(L, \mathbb{Z})$. The set of Bohr-Sommerfeld leaves $\mathscr{L}_{B S}$ inherits the structure of topological groupoid. As a consequence of the above properties, $\mathscr{L}_{B S}$ is equipped with a groupoid one cocycle (the modular cocycle) and a two cocycle (the prequantization cocycle). In the next section we will see what kind of regularity we require in order to extract from these data a suitable quantization.

\section{Haar systems}

The definition of Haar system for a groupoid extends the Haar measure on groups. In the presentation we follow [ $[$ ] (see also [ $[8]$ ).

Let $\mathscr{G}$ be a locally compact groupoid (for simplicity we assume that it is Hausdorff) and let $C_{c}(\mathscr{G})$ denote the space of continuous functions with compact support. A left Haar system for $\mathscr{G}$ is a family of measures $\left\{\lambda^{x}, x \in \mathscr{G}_{0}\right\}$ on $\mathscr{G}$ such that

i) the support of $\lambda^{x}$ is $\mathscr{G}^{x}=l_{\mathscr{G}}^{-1}(x)$;

ii) for any $f \in C_{c}(\mathscr{G})$, and $x \in \mathscr{G}_{0}, \lambda(f)(x)=\int_{\mathscr{G}} f d \lambda^{x}$, defines $\lambda(f) \in C_{c}\left(\mathscr{G}_{0}\right)$; 
iii) for any $\gamma \in \mathscr{G}$ and any $f \in C_{c}(\mathscr{G}), \int_{\mathscr{G}} f\left(\gamma \gamma^{\prime}\right) d \lambda^{r_{\mathscr{G}}(\gamma)}\left(\gamma^{\prime}\right)=\int_{\mathscr{G}} f\left(\gamma^{\prime}\right) d \lambda^{\lg _{\mathscr{G}}}(\gamma)\left(\gamma^{\prime}\right)$.

The existence of the Haar system imposes a topological constraint on $\mathscr{G}$, in particular in [Q] it is proven that as a consequence the right map $r \mathscr{G}: \mathscr{G} \rightarrow \mathscr{G}_{0}$ is an open map. Moreover, if it exists it is not unique, in general. There are two cases where there is a canonical choice, Lie groupoids and $r$-discrete groupoids. In Lie case, the Haar system is unique once one adds a smoothness condition (see Chapter 2.3 in [8]]). A locally compact groupoid $\mathscr{G}$ is $r$-discrete if $r_{\mathscr{G}}: \mathscr{G} \rightarrow \mathscr{G}_{0}$ is a local homeomorphism. As a consequence the fibre of $r_{\mathscr{G}}$ is dicrete and admits the counting measure as the essentially unique Haar measure. It is also called an étale groupoid.

The composition of $\lambda^{x}$ with the inverse map will be denoted as $\lambda_{x}$; this family defines a right Haar system $\lambda^{-1}$. Any measure $\mu$ on the space of units $\mathscr{G}_{0}$ induces measures $v, v^{-1}$ on the whole $\mathscr{G}$ through

$$
\int_{\mathscr{G}} f d v=\int \lambda(f) d \mu, \quad \int_{\mathscr{G}} f d v^{-1}=\int \lambda^{-1}(f) d \mu .
$$

The measure $\mu$ is said to be quasi-invariant if $v$ and $v^{-1}$ are equivalent measures; in this case the Radon-Nikodym derivative $D=d v / d v^{-1}$ is called the modular function of $\mu$. The function $\log D \in Z^{1}(\mathscr{G}, \mathbb{R})$ turns out to be a groupoid one cocycle with values in $\mathbb{R}$ and its cohomology class depends only on the equivalence class of $\mu$.

The notion of quasi-invariant measure can be extended as follows: let $c \in Z^{1}(\mathscr{G}, \mathbb{R})$ be a fixed cocycle and $\beta \in[-\infty, \infty]$. Consider the set:

$$
\operatorname{Min}(c)=\left\{x \in \mathscr{G}_{0}: c\left(\mathscr{G}_{x}\right) \subset[0, \infty)\right\} .
$$

A measure $\mu$ on $\mathscr{G}_{0}$ satisfies the $(c, \beta)-K M S$ condition if

$i$ ) when $\beta$ is finite, $\mu$ is quasi invariant and the modular function is $e^{-\beta c}$.

ii) when $\beta= \pm \infty$, the support of $\mu$ is contained in $\operatorname{Min}( \pm c)$.

A normalized $(c, \infty)$-KMS measure is called a ground state for $c$.

Let $\zeta \in Z^{2}(\mathscr{G}, \mathbb{T})$ be a continuous two-cocycle. For any $f, g \in C_{c}(\mathscr{G})$ let us define the convolution and the involution as

$$
\begin{aligned}
(f * g)(\gamma) & :=\int f\left(\gamma \gamma^{\prime}\right) g\left(\gamma^{-1}\right) \zeta\left(\gamma \gamma^{\prime}, \gamma^{-1}\right) d \lambda^{r_{g}(\gamma)}\left(\gamma^{\prime}\right), \\
f^{*}(\gamma) & :=\overline{f\left(\gamma^{-1}\right)} \overline{\zeta\left(\gamma, \gamma^{-1}\right)}
\end{aligned}
$$

The space $C_{c}(\mathscr{G})$ equipped with these operations defines the $*$-algebra $C_{c}(\mathscr{G}, \zeta)$. The $C^{*}$-norm is defined as $\|f\|=\sup _{L}|| L(f)||$ over all bounded representations $L$ (we skip details, see [Q]]). The completion of $C_{c}(\mathscr{G}, \zeta)$ with respect to this norm defines the $\zeta$-twisted convolution $C^{*}$-algebra $C^{*}(\mathscr{G}, \zeta)$ of the groupoid $\mathscr{G}$. We omit $\zeta$ in the notation when the cocycle is the trivial one.

Let now $c \in Z^{1}(\mathscr{G}, \mathbb{R})$ be a one cocycle with values in $\mathbb{R}$. It defines an algebra automorphism $A_{c}: \mathbb{R} \rightarrow \operatorname{Aut}\left(C_{c}(\mathscr{G}, \zeta)\right)$ as

$$
\left(A_{c}(t) f\right)(\gamma)=e^{i t c(\gamma)} f(\gamma) \quad f \in C_{c}(\mathscr{G}, \zeta) .
$$

A measure $\mu$ on the space of units $\mathscr{G}_{0}$ defines the weight $\phi_{\mu}$ on $C^{*}(\mathscr{G}, \zeta)$ as

$$
\phi_{\mu}(f)=\int_{\mathscr{G}_{0}} f d \mu
$$


We recall that given the automorphism $A_{c}$ the weight $\phi_{\mu}$ satisfies the KMS condition at $0 \leq \beta<\infty$ if, for any $f, g \in C_{c}(\mathscr{G}, \zeta)$, we have

$$
\phi_{\mu}\left(f * A_{c}(i \beta)(g)\right)=\phi_{\mu}(g * f) \quad ;
$$

if $\beta=\infty, \phi_{\mu}$ satisfies the KMS condition at $\infty$ if $\left|\phi_{\mu}\left(f * A_{c}(z)(g)\right)\right| \leq\|f\|\|g\|$, for any $z$ such that $\operatorname{Im} z>0$. It is proven that $\mu$ satisfies the $(c, \beta)-\mathrm{KMS}$ condition for $\beta \in[0, \infty]$ if and only if $\phi_{\mu}$ satisfies the KMS condition for the automorphism $A_{c}$ at $\beta$.

The GNS representation generated by the KMS weight $\phi_{\mu}$ is obtained as convolution action of $C_{c}(\mathscr{G}, \zeta)$ on the Hilbert space $L^{2}\left(\mathscr{G}, v^{-1}\right)$. If $\beta$ is finite (and so $\mu$ is quasi invariant and $D=e^{-\beta c}$ is the modular function) then $C_{c}(\mathscr{G}, \zeta)$, equipped with the inner product of $L^{2}\left(\mathscr{G}, v^{-1}\right)$, is a left Hilbert algebra. We recall the definition.

Definition 4.1. A *-algebra $\mathscr{A}$, equipped with a inner product, is a left Hilbert algebra if the left regular representation is bounded and involutive, if $S(f)=f^{*}, f \in \mathscr{A}$, is preclosed, and if $\mathscr{A}^{2}$ is dense in $\mathscr{A}$.

The polar decomposition of $S=J D^{1 / 2}$ defines the modular conjugation $J$ and the modular operator $D=S^{\dagger} S$. On $f \in C_{c}(\mathscr{G}, \zeta)$ we have

$$
(J f)(\gamma)=D^{1 / 2}(\gamma) f^{*}(\gamma), \quad(D f)(\gamma)=D(\gamma) f(\gamma) .
$$

In case $\beta=\infty$ the KMS condition means that the hamiltonian $c$ is positive on $\operatorname{supp}(v)$ and the associated state $\phi_{\mu}$ is called a ground state.

We are now in a position to formulate our general philosophy of the quantization by means of a multiplicative real polarization (in particular of a multiplicative integrable model).

In the previous section we saw that such a polarization will produce the topological groupoid $\mathscr{L}_{B S}$ of Bohr-Sommerfeld leaves, equipped with the modular one cocycle $F \in Z^{1}\left(\mathscr{L}_{B S}, \mathbb{R}\right)$ and the prequantization two cocycle $\zeta \in Z^{2}\left(\mathscr{L}_{B S}, \mathbb{T}\right)$. We require that the groupoid of Bohr-Sommerfeld leaves $\mathscr{L}_{B S}$ is $r$-discrete, so that there exists a canonical Haar system. If this condition is satisfied we can apply Renault's construction briefly described in this Section. Let $\mu_{F}$ be the quasi invariant measure determined by the modular cocycle $F$ and $v$ the induced measure on $\mathscr{L}_{B S}$. The ouptut of the quantization procedure is then the left Hilbert algebra $C_{c}\left(\mathscr{L}_{B S}, \zeta\right)$ endowed with the scalar product of $L^{2}\left(\mathscr{L}_{B S}, v^{-1}\right)$.

\section{A multiplicative integrable model for the Podles sphere}

The standard Podles sphere $\left(\mathbb{S}^{2}, \pi\right)$ is the simplest example of Poisson homogeneous space for Poisson-Lie groups. It is a Poisson manifold with a two symplectic leaves: the north pole $\{\infty\}$ is the zero dimensional leaf and the complement $\mathbb{S}^{2} \backslash\{\infty\}$ is the symplectic one. We call $\mathbb{S}^{2} \backslash\{\infty\}$ the symplectic chart with coordinate $z$ and $\mathbb{S}^{2} \backslash\{z=0\}$ the singular chart with coordinate $w=1 / z$. In the symplectic chart, the Poisson bracket reads

$$
\{z, \bar{z}\}=i\left(1+|z|^{2}\right) \quad .
$$


One can check that this bracket extends to the other chart $w=1 / z$ and that $w=0$ is a zero for the Poisson tensor of degree 2.

Let us describe very briefly how its symplectic groupoid is defined. Details can be found in [四]. As a manifold, the symplectic groupoid $\mathscr{G}\left(\mathbb{S}^{2}, \pi\right)$ is $T^{*} \mathbb{S}^{2}$. In the complex coordinates $(z, p)$ on the symplectic chart the structure maps read

$$
\begin{gathered}
l_{\mathscr{G}}(z, p)=z, \quad r_{\mathscr{G}}(z, p)=z+\left(1+|z|^{2}\right) \bar{p}, \\
m_{\mathscr{G}}\left[(z, p)\left(z^{\prime}, p^{\prime}\right)\right]=\left(z, p+\frac{1+\left|z^{\prime}\right|^{2}}{1+|z|^{2}}\right), \quad z^{\prime}=r_{\mathscr{G}}(z, p) .
\end{gathered}
$$

We can use the fact the symplectic leaf is open an write $\left.\mathscr{G}\left(\mathbb{S}^{2}, \pi\right)\right|_{\left.\mathbb{S}^{2}\right\rangle_{\infty}}$ as $\mathbb{C} \times \mathbb{C}$, where $(x, y) \in$ $\mathbb{C} \times \mathbb{C}$ are defined as $x=l_{\mathscr{G}}(z, p)$ and $y=r_{\mathscr{G}}(z, p)$. The symplectic form is $\Omega_{\mathscr{G}}=l_{\mathscr{G}}^{*} \omega-r_{\mathscr{G}}^{*} \omega$, where $\omega=i d z d \bar{z} /\left(1+|z|^{2}\right)$ is the symplectic form on $\mathbb{S}^{2} \backslash\{\infty\}$; it can be explicitly checked that it extends to the singular chart and defines a global form.

Up to a scalar multiplication, any Haar system on $\mathscr{G}\left(\mathbb{S}^{2}, \pi\right)$ is written as

$$
\lambda^{x}=-i \Lambda(y) d y d \bar{y},(|x|<\infty) ; \quad \lambda^{\infty}=-i d p_{S} d \bar{p}_{S},
$$

for any positive $\Lambda$ such that $\lim _{y \rightarrow \infty} \Lambda(y)=1$ ( $p_{S}$ is the momentum of $w$ in the singular chart). The modular function with respect to the round volume form on $\mathbb{S}^{2}$ is

$$
F(x, y)=\log \frac{1+|x|^{2}}{1+|y|^{2}} .
$$

Since the symplectic groupoid is diffeomorphic to the cotangent bundle with an exact symplectic form (but not the canonical one) the prequantization is just given by the trivial line bundle $\Lambda=T^{*} \mathbb{S}^{2} \times \mathbb{C}$ equipped with the connection given by choosing a primitive $\Theta_{\mathscr{G}}$ of $\Omega \mathscr{G}$.

Let now $\tau=\frac{1}{1+|z|^{2}}$; remark that $\tau \in C^{\infty}\left(\mathbb{S}^{2}\right)$, i.e. it is a global function. Then the two hamiltonians $F_{1}=l_{\mathscr{G}}^{*}(\tau)$ and $F_{2}=r_{\mathscr{G}}^{*}(\tau)$ are in involution because $l_{\mathscr{G}}$ and $r_{\mathscr{G}}$ are symplectically orthogonal. Moreover, the modular function is $F=\log l_{\mathscr{G}}^{*}(\tau) / r_{\mathscr{G}}^{*}(\tau)$, so that we have a multiplicative integrable system.

The Bohr-Sommerfeld leaves are selected by the condition

$$
\int_{|x|^{2}=F_{1}} \Theta_{\mathscr{G}}=2 \pi n_{1} \hbar, \quad \int_{|y|^{2}=F_{2}} \Theta_{\mathscr{G}}=2 \pi n_{2} \hbar \quad n_{1}, n_{2} \in \mathbb{Z} .
$$

The groupoid of BS leaves can be mapped to the subgroupoid $\mathscr{L}_{B S}=\{(m, n), m+n \geq 0, n \geq 0\} \cup$ $\left.\{\infty\} \subset(\mathbb{Z} \times \overline{\mathbb{Z}})\right|_{\bar{N}}$, with $n=n_{1}$ and $m=n_{2}-n_{1}$. The groupoid structure is the one induced by the action groupoid $\mathbb{Z} \times \overline{\mathbb{Z}}$, defined by the action of $\mathbb{Z}$ on $\overline{\mathbb{Z}}=\mathbb{Z} \cup\{\infty\}$. The groupoid $\mathscr{L}_{B S}$ is $r$ discrete so that there exists a canonical left Haar system given by the counting measure. It is the groupoid appearing in Sheu's description of the $C^{*}$-algebra of the quantum Podles̀ sphere in [U]]. The modular cocycle on the Bohr-Sommerfeld groupoid reads as $F(m, n)=\hbar m$. The corresponding normalized quasi-invariant measure on $\left(\mathscr{L}_{B S}\right)_{0}=\overline{\mathbb{N}}$ reads $\mu_{\hbar}(n)=e^{-n \hbar}\left(1-e^{-\hbar}\right)$. The convolution algebra is generated by $\left\{e_{m, n}, n \geq 0, m+n \geq 0\right\}$ and the identity id $=\sum_{n \geq 0} e_{0, n}$ satisfying

$$
e_{m n} * e_{m^{\prime} n^{\prime}}=\delta_{n, m^{\prime}+n^{\prime}} e_{m+m^{\prime}, n^{\prime}} \quad, \quad\left(e_{m n}, e_{m^{\prime} n^{\prime}}\right)=\delta_{m m^{\prime}} \delta_{n n^{\prime}} \mu_{\hbar}(n)
$$


In [2] it is introduced also a complex polarization so that the general construction of geometric quantization can be applied. The Hilbert algebra described in (5.2) can be recovered from the definition of a convolution product on the space of polarized sections. We want to remark that the output of quantization obtained following the groupoid $C^{*}$-algebra procedure is the convolution algebra defined in the geometric quantization framework.

\section{References}

[1] S. Bates, A. Weinstein, Lectures on the geometry of quantization. American Mathematical Society, Berkeley Mathematical Lecture Notes, 8, (1997).

[2] F. Bonechi, N. Ciccoli, N. Staffolani, M. Tarlini, The quantization of the symplectic groupoid of the standard Podles̀ sphere. [arXiv:math.SG/1004.3163], Journal of Geometry and Physics, at press.

[3] F. Bonechi, N. Ciccoli, M. Tarlini, A multiplicative integrable model for Poisson-Lie groups, in preparation.

[4] A.S. Cattaneo and G. Felder, Poisson sigma models and symplectic groupoids. In "Quantization of Singular Symplectic Quotients”, (ed. N. P. Landsman, M. Pflaum, M. Schlichenmeier), Progress in Mathematics, 198 (2001) 41-73.

[5] M. Crainic, Prequantization and Lie brackets. J. Symplectic Geom., 2 (2004) 579-602 [arXiv:math.DG/0403269].

[6] M. Crainic and R. L. Fernandes, Integrability of Lie brackets. Ann. of Math., 157 (2003) 575-620.

[7] E. Hawkins, A groupoid approach to quantization. J. Symplectic Geom., 6 (2008) 61-125 [arXiv:math.SG/0612363].

[8] A. L. T. Paterson, Groupoids, inverse semigroups, and their operator algebras. Progress in Mathematics, 170 Birkhäuser (1999).

[9] J. Renault, A groupoid approach to $C^{*}$-algebras. Lectures Notes in Mathematics 793, Springer Verlag (1980).

[10] A.J.L. Sheu, Quantum Spheres As Groupoid $C^{*}$-algebras. The Quarterly Journal of Mathematics, 48 (1997) 503-510

[11] A.J.L. Sheu, Groupoid Approach to Quantum Projective Spaces. Contemporary Mathematics, 228 (1998) 341-350 [arXiv:math/9802083v1]

[12] A. Weinstein, Symplectic groupoids and Poisson mnifolds. Bulletin of the American Mathematical Society, 161 (1987), 101-104

[13] A. Weinstein, Symplectic groupoids, geometric quantization and irrational rotation algebras. In "Symplectic geometry, groupoids and integrable systems" (Berkeley, CA, 1989), 281-290, Springer, New York (1991). MR1104934.

[14] A. Weinstein and P. Xu, Extensions of symplectic groupoids and quantization. J. Reine Angew. Math., 417 (1991) 159-189

[15] A. Weinstein, The modular automorphism of a Poisson manifold. Journal of Geometry and Physics, 23 (1997) 379-394. 